\title{
Complement-independent retinal pathology produced by intravitreal injection of neuromyelitis optica immunoglobulin $\mathrm{G}$
}

\author{
Christian M. Felix ${ }^{1,2}$, Marc H. Levin ${ }^{1,3+}$ and Alan S. Verkman ${ }^{2^{*}+}$
}

\begin{abstract}
Background: Neuromyelitis optica (NMO), an autoimmune inflammatory disease of the central nervous system, is often associated with retinal abnormalities including thinning of the retinal nerve fiber layer and microcystic changes. Here, we demonstrate that passive transfer of an anti-aquaporin-4 autoantibody (AQP4-lgG) produces primary retinal pathology.

Methods: AQP4-lgG was delivered to adult rat retinas by intravitreal injection. Rat retinas and retinal explant cultures were assessed by immunofluorescence.

Results: Immunofluorescence showed AQP4-IgG deposition on retinal Müller cells, with greatly reduced AQP4 expression and increased glial fibrillary acidic protein by 5 days. There was mild retinal inflammation with microglial activation but little leukocyte infiltration and loss of retinal ganglion cells by 30 days with thinning of the ganglion cell complex. Interestingly, the loss of AQP4 was complement independent as seen in cobra venom factor-treated rats and in normal rats administered a mutated AQP4-lgG lacking complement effector function. Exposure of ex vivo retinal cultures to AQP4-lgG produced a marked reduction in AQP4 expression by $24 \mathrm{~h}$, which was largely prevented by inhibitors of endocytosis or lysosomal acidification.
\end{abstract}

Conclusions: Passive transfer of AQP4-IgG results in primary, complement-independent retinal pathology, which might contribute to retinal abnormalities seen in $\mathrm{NMO}$ patients.

Keywords: NMO, Retina, Müller cells, Aquaporin-4, Complement, Autoimmunity

\section{Background}

Neuromyelitis optica (NMO) is an autoimmune inflammatory disease of the central nervous system (CNS) that causes optic neuritis and transverse myelitis, leading to loss of vision and motor function, reviewed in [1-4]. Affected tissues in NMO generally show astrocyte damage with complement activation, inflammatory cell infiltration, and demyelination. Most NMO patients are seropositive for immunoglobulin $\mathrm{G}$ autoantibodies against aquaporin-4 (AQP4), a water channel expressed

\footnotetext{
* Correspondence: Alan.Verkman@ucsf.edu; http://www.ucsf.edu/verklab ${ }^{\dagger}$ Equal contributors

${ }^{2}$ Departments of Medicine and Physiology, University of California, San Francisco, 1246 Health Sciences East Tower, San Francisco, CA 94143-0521, USA

Full list of author information is available at the end of the article
}

on astrocytes. Human NMO pathology and rodent models of NMO produced by passive transfer of antiAQP4 autoantibody (AQP4-IgG) suggest a pathogenesis mechanism in which AQP4-IgG binding to AQP4 causes primary complement- and cell-mediated astrocyte toxicity, with a secondary inflammatory response leading to oligodendrocyte injury, demyelination, and axon loss.

NMO patients often manifest retinal abnormalities including thinning of the retinal nerve fiber layer (RNFL) and ganglion cell layer (GCL) following episodes of optic neuritis [5-7]. During acute episodes of NMO optic neuritis, the inner nuclear layer (INL) typically thickens $[8,9]$, often coincident with inner retinal microcystic changes $[10,11]$. It has been speculated that retinal injury in NMO may in part be a primary disease 
manifestation, although it has been difficult to resolve direct retinal injury from retrograde axon degeneration due to retrobulbar optic neuritis. These retinal abnormalities are also seen, albeit at lower frequency, following optic neuritis in multiple sclerosis and a variety of severe noninflammatory optic neuropathies [12-15].

The cellular makeup and structure of the retina is quite different from the optic nerve, spinal cord, and brain, where there are myelinated nerve fibers and AQP4 expression on astrocytes [16]. In the retina, AQP4 is expressed in two types of retinal glia: Müller cells, which are a specialized form of radial glia spanning the retinal inner limiting to outer limiting membranes, with the cell body lying within the INL, and astrocytes, which are mostly localized in the RNFL along with nonmyelinated axons of retinal ganglion cells (RGCs).

Here, to investigate the possibility that NMO autoantibodies could produce primary retinal injury, we exposed rat retinas to AQP4-IgG in vivo by intravitreal injection. Rats were chosen because they have human-like serum complement activity [17] and have been used in various models in which AQP4-IgG produces characteristic NMO pathology in CNS tissues [18, 19]. We found that intravitreally delivered AQP4-IgG efficiently bound to AQP4 on retinal Müller cells and, unexpectedly, produced unique complement-independent retinal injury, which was further characterized by studies on ex vivo retinal cultures exposed to AQP4-IgG.

\section{Methods}

\section{Rats}

Adult male Sprague-Dawley rats (age 8-10 weeks) were purchased from Charles River Laboratories (Wilmington, MA). $\mathrm{CD} 59^{-1-}$ rats were generated using CRISPR-Cas9 technology, which will be reported separately. Protocols were approved by the University of California San Francisco Committee on Animal Research and were in compliance with the ARVO Statement for the Use of Animals in Ophthalmic and Visual Research.

\section{Intravitreal injections}

Rats were anesthetized using $3 \%$ isoflurane and topical proparacaine ( $0.5 \%$, Akorn, Lake Forest, IL), and their pupils were dilated with phenylephrine $(2.5 \%$, Paragon BioTeck Inc., Portland, OR) and atropine (1\%, Akorn). In each eye, a full-thickness track was made through superotemporal sclera at the pars plana with a 30-gauge needle for intravitreal delivery of a $4-\mu \mathrm{L}$ solution volume using a 33-gauge beveled needle attached to a $10-\mu \mathrm{L}$ Hamilton syringe (Reno, NV). Microscopic examination was performed to confirm the absence of vitreous hemorrhage or gross lens trauma. Lubricating ophthalmic ointment was applied to protect the cornea until recovery from anesthesia.
Both eyes in each rat received the same treatment to control for potential contralateral effects of a given treatment. The following experimental groups were evaluated: (i) no injection, or injection of (ii) saline; (iii) control human IgG (control-IgG; $40 \mu \mathrm{g}$; Pierce Biotechnology, Rockford, IL); (iv) purified human monoclonal recombinant AQP4-IgG (rAb-53, $40 \mu \mathrm{g}$, ref. [20]); (v) recombinant monoclonal antiAQP4 "aquaporumab"-lacking effector functions (AQP4-IgG-CDC, $4 \mu \mathrm{g}$, ref. [21]); or (vi) lipopolysaccharide (LPS) from Escherichia coli $(5 \mu \mathrm{g}$; SigmaAldrich, St. Louis, MO). In some experiments, rat complement was inactivated by intraperitoneal injection of cobra venom factor $(\mathrm{CVF} ; 600 \mathrm{U} / \mathrm{kg}$, Quidel Corporation, Santa Clara, CA) $24 \mathrm{~h}$ before and $48 \mathrm{~h}$ after intravitreal injection of AQP4-IgG, as described in [19]. Rats were sacrificed 6 h, 24 h, 5 days, or 30 days after intravitreal injection. Globes were enucleated after transcardiac perfusion with phosphate-buffered saline (PBS) followed by $4 \%$ paraformaldehyde, fixed for $4 \mathrm{~h}$ and left overnight at $4{ }^{\circ} \mathrm{C}$ in $30 \%$ sucrose. The eyes were embedded in OCT and sectioned axially at $20-\mu \mathrm{m}$ thickness.

\section{Retinal explant cultures}

Rats were deeply anesthetized with isoflurane and then decapitated. The freshly enucleated eyes were immersed in ice-cold Hank's balanced salt solution (HBSS) containing $1 \%$ penicillin-streptomycin. Using a dissecting microscope, a circumferential incision was made at the pars plana, followed by removal of the anterior segment, lens, and vitreous body. With Dumont forceps, the retinas were separated from the sclera and separated from the optic nerve head. Each retina was cut radially and separated into four quadrants, which were each transferred with inner retinal surfaces facing up onto $12-\mathrm{mm}$ diameter filters $(0.4-\mu \mathrm{m}$ pore; Sigma-Aldrich) in 12-well plates.

Retinal explants were maintained immersed in a thin layer of serum-free culture medium at an air/medium interface in a $5 \% \mathrm{CO}_{2}$ incubator at $37^{\circ} \mathrm{C}$. Culture media contained neuronal growth medium (Neurobasal A) supplemented with $2 \% \mathrm{~B} 27,1 \% \mathrm{~N} 2$, L-glutamine $(0.8 \mathrm{mM})$, and $1 \%$ penicillin-streptomycin. One half of the media was replaced after $24 \mathrm{~h}$ in culture. AQP4-IgG (final $20 \mu \mathrm{g} / \mathrm{mL}$ ) was added to some wells after the initial $24 \mathrm{~h}$ in culture. Some explants were also incubated with dynasore hydrate (inhibitor of dynamin-dependent endocytosis; $50 \mu \mathrm{M}$ ) or chloroquine (inhibitor of lysosomal degradation; $10 \mu \mathrm{M})$. At $24 \mathrm{~h}$ later, explants were fixed in $4 \%$ PFA for $24 \mathrm{~h}$ and then placed in $30 \%$ sucrose for $24 \mathrm{~h}$ at $4{ }^{\circ} \mathrm{C}$ before embedding in OCT. Sections were cut at $10-\mu \mathrm{m}$ thickness perpendicular to the fullthickness retina. 


\section{Immunofluorescence}

Frozen sections were incubated in blocking solution (PBS, 1 \% bovine serum albumin, $0.2 \%$ Triton $\mathrm{X}-100$ ) for $1 \mathrm{~h}$ prior to overnight incubation $\left(4^{\circ} \mathrm{C}\right)$ with primary antibodies against the following: AQP4 (1:200, Santa Cruz Biotechnology, Santa Cruz, CA), glial fibrillary acidic protein (GFAP, 1:100, Millipore), glutamine synthetase (GS, 1:500, Sigma-Aldrich), Brn3a (1:100, Santa Cruz Biotechnology), ionized calcium-binding adaptor molecule-1 (Iba1; 1:1000; Wako, Richmond, VA), C1q (1:50, Abcam, Cambridge, MA), or C5b-9 (1:50, Hycult Biotech, Uden, Netherlands), followed by appropriate species-specific Alexa Fluor-conjugated secondary antibody for $1 \mathrm{~h}$ at room temperature (1:200, Invitrogen, Carlsbad, CA). Rinsed sections were mounted with VECTASHIELD with 4,6-diamidino-2-phenylindole (DAPI) (Vector Laboratories, Burlingame, CA). Staining with hematoxylin and eosin ( $\mathrm{H} \& \mathrm{E})$ was done using standard procedures. Sections were visualized on a Leica epifluorescence microscope (Wetzlar, Germany) or Nikon confocal fluorescence microscope (Melville, NY).

AQP4, GFAP, and Iba1 immunofluorescence were quantified in $\times 20$ fields of central retina, $50 \mu \mathrm{m}$ from the optic nerve head. AQP4 and GFAP fluorescence were defined using the polygon drawing tool and quantified using ImageJ (NIH, Bethesda, MD). For AQP4 quantification, retinal layers were segmented into RNFL + GCL, inner plexiform layer (IPL) + INL, and outer plexiform layer $(\mathrm{OPL})+$ outer nuclear layer $(\mathrm{ONL})$. GFAP was measured in two segments: (RNFL + GCL) and (IPL + $\mathrm{INL}+\mathrm{OPL}+\mathrm{ONL})$. Data are presented as a percentage of area of immunofluorescence loss normalized to untreated retinas.

RGCs were counted at day 30 after intravitreal injection of AQP4-IgG or control-IgG as the density of Brn3a-positive nuclei in fluorescence micrographs of retinal flat mounts. After transcardiac perfusion, the eyes were enucleated and the retinas were removed and immunostained in culture wells on a shaker. Four radial relaxing incisions were made, and the retinas were flattened and coverslipped with VECTASHIELD mounting media. A total of 12 nonoverlapping images $(\times 20$ magnification), each including nonoverlapping posterior, middle, or anterior retina of one quadrant, were taken using the epifluorescence microscope. Brn3a-positive nuclei were counted semi-automatically using cell-counting plugins from ImageJ software.

\section{Optical coherence tomography and fundoscopy}

Rats were induced and maintained under isoflurane gas anesthesia, and the eyes were numbed and dilated as before. The corneas were lubricated with $2.5 \%$ Goniovisc (Accutome Inc., Malvern, PA) for direct contact with the imaging lens. The eyes were examined using the Micron
III retinal imaging system (Phoenix Research Labs, Pleasanton, CA), and raw fundus photographs were captured. Spectral-domain optical coherence tomography (SD-OCT) horizontal line scans were acquired on a micron image-guided SD-OCT system (Phoenix Research Labs) by averaging 10 scans. Potential inner retinal atrophy was evaluated in vivo as longitudinal changes in thickness of the ganglion cell complex (GCC, encompassing RNFL, GCL, and IPL). GCC and total retinal thicknesses at single points $1500 \mu \mathrm{m}$ from the nasal and temporal optic nerve head margins were averaged as a single point per eye.

\section{Statistics}

Data are presented as mean \pm S.E.M. The two-sided Student's $t$ test was used for direct comparisons between two means. When there were three or more groups, analysis was done using a one-way ANOVA with Dunnett post hoc test. Analysis was performed using Prism 5 GraphPad Software package (GraphPad Software, San Diego, CA). Significance levels were set at $p<0.05{ }^{(*)}$ and $p<0.01{ }^{(* *)}$.

\section{Results}

Intravitreal AQP4-IgG reduces Müller cell AQP4 expression and causes reactive gliosis

As diagrammed in Fig. 1a, rats were administered AQP4-IgG (or control human IgG) by intravitreal injection and then sacrificed at different times. The injected eyes were grossly normal at sacrifice, without uveitis or hemorrhage, and only rare focal lens opacifications. En face immunofluorescence of retinal flat mounts showed AQP4-IgG deposition on the retina in a membrane pattern at 3 days after injection, as seen using a secondary antibody against human IgG, with loss of AQP4 immunofluorescence (Fig. 1b). Human IgG binding was not seen in astrocytes along myelinated optic nerves (not shown), demonstrating selective exposure of the retina to AQP4-IgG.

Immunofluorescence of frozen sections of the posterior retina showed AQP4-IgG localization in a pattern corresponding to Müller cells (Fig. 1c). There was some loss of AQP4 immunofluorescence by $6 \mathrm{~h}$, which became more marked at $24 \mathrm{~h}$ and 5 days but largely returned to initial levels by 30 days. Also notable was Müller cell gliosis, seen as an upregulation of GFAP expression most evident at 5 days, as well as a deposition of activated complement in an apparent perivascular pattern seen best at $24 \mathrm{~h}$ and 5 days. Quantification of retinal AQP4 and GFAP immunofluorescence is summarized in Fig. 1d.

At higher magnification, AQP4 immunofluorescence colocalized with the Müller cell marker GS (Fig. 2a). GS immunofluorescence was stable even after AQP4 was 

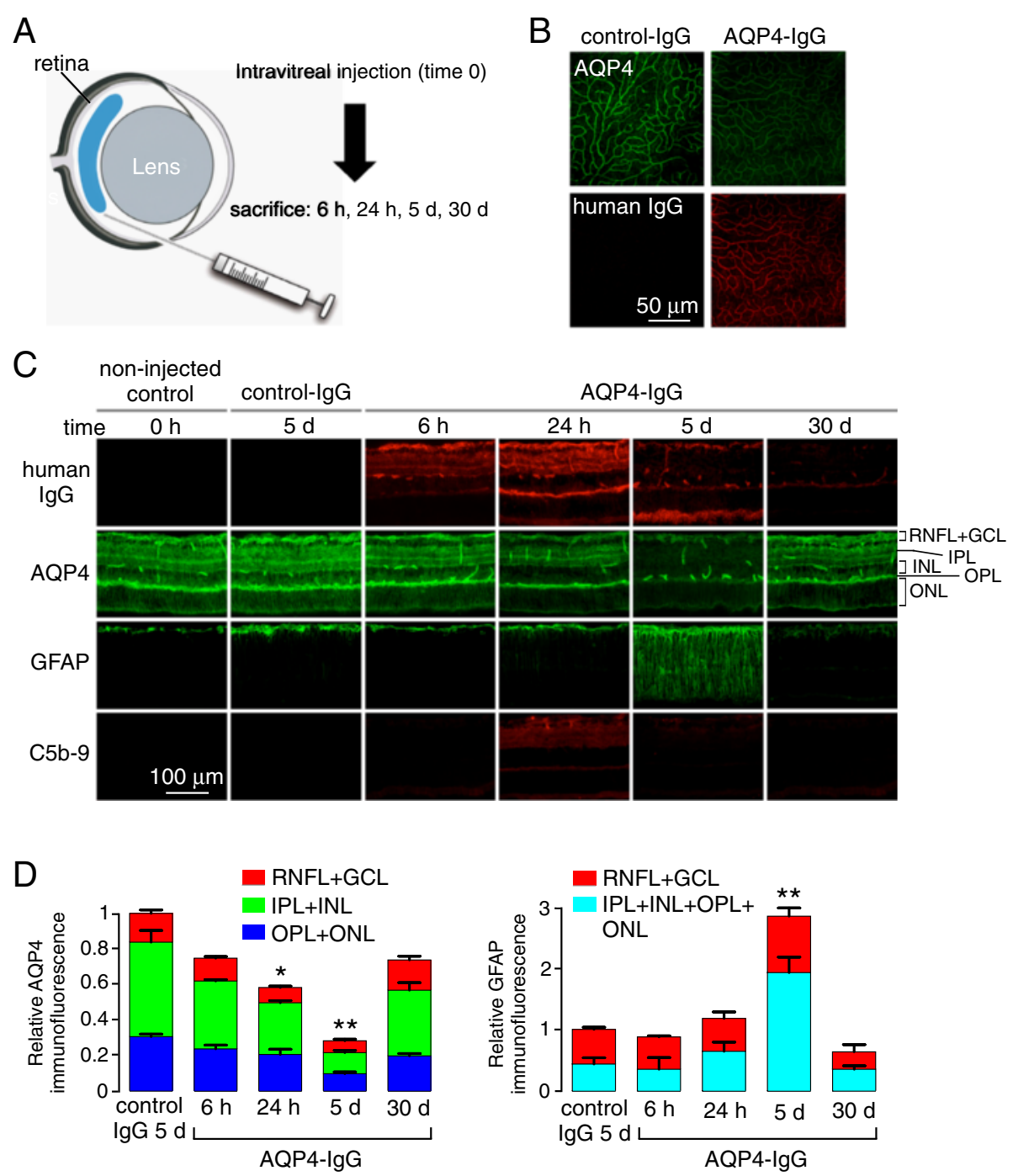

Fig. 1 Consequences of intravitreal administration of AQP4-IgG in rat. a Injection approach. b AQP4 and human IgG immunofluorescence in retinal flat mounts 5 days after intravitreal delivery of $40 \mu \mathrm{g}$ control human IgG or AQP4-lgG in rat. c Immunofluorescence on transverse retinal sections for experiments as in (b). Representative of 4 eyes per condition. $\mathbf{d}$ Relative immunofluorescence of AQP4 (left) and GFAP (right) in indicated retinal layers from sections as in (c) (mean \pm S.E.M, 2 rats, 4 eyes per condition, ${ }^{*} p<0.05,{ }^{* *} p<0.01$ )

greatly reduced, suggesting that the Müller cells remained viable. As expected, intravitreally delivered AQP4-IgG also localized to cell membranes of nonpigmented ciliary epithelia, the other major ocular site of AQP4 expression (Fig. 2b). In contrast to the retina, AQP4-IgG deposition in the ciliary body did not cause loss of AQP4 at 5 days after administration nor was there demonstrable inflammatory infiltrates as seen by H\&E staining.

\section{Loss of Müller cell AQP4 following intravitreal AQP4-IgG is complement independent}

A large body of data in human NMO and in animal models implicates complement deposition and activation on AQP4-expressing astrocytes in the brain and spinal cord in disease pathogenesis. To investigate whether complement activation is required to produce AQP4 loss following intravitreal AQP4-IgG injection, studies were done in rats treated with CVF, which inactivates complement and was shown previously to prevent NMO pathology following intracerebral injection of AQP4-IgG in rats [19]. At 5 days following intravitreal injection of AQP4-IgG, the CVF-treated rats showed similar loss of Müller cell AQP4 as seen in the untreated rats, as well as comparable Müller cell gliosis (Fig. 3a), suggesting that these retinal changes are complement independent. Also in support of this conclusion was the finding that delivery of an engineered AQP4-IgG lacking complement effector function (in place of AQP4-IgG) produced similar AQP4 loss and Müller cell gliosis. Moreover, 


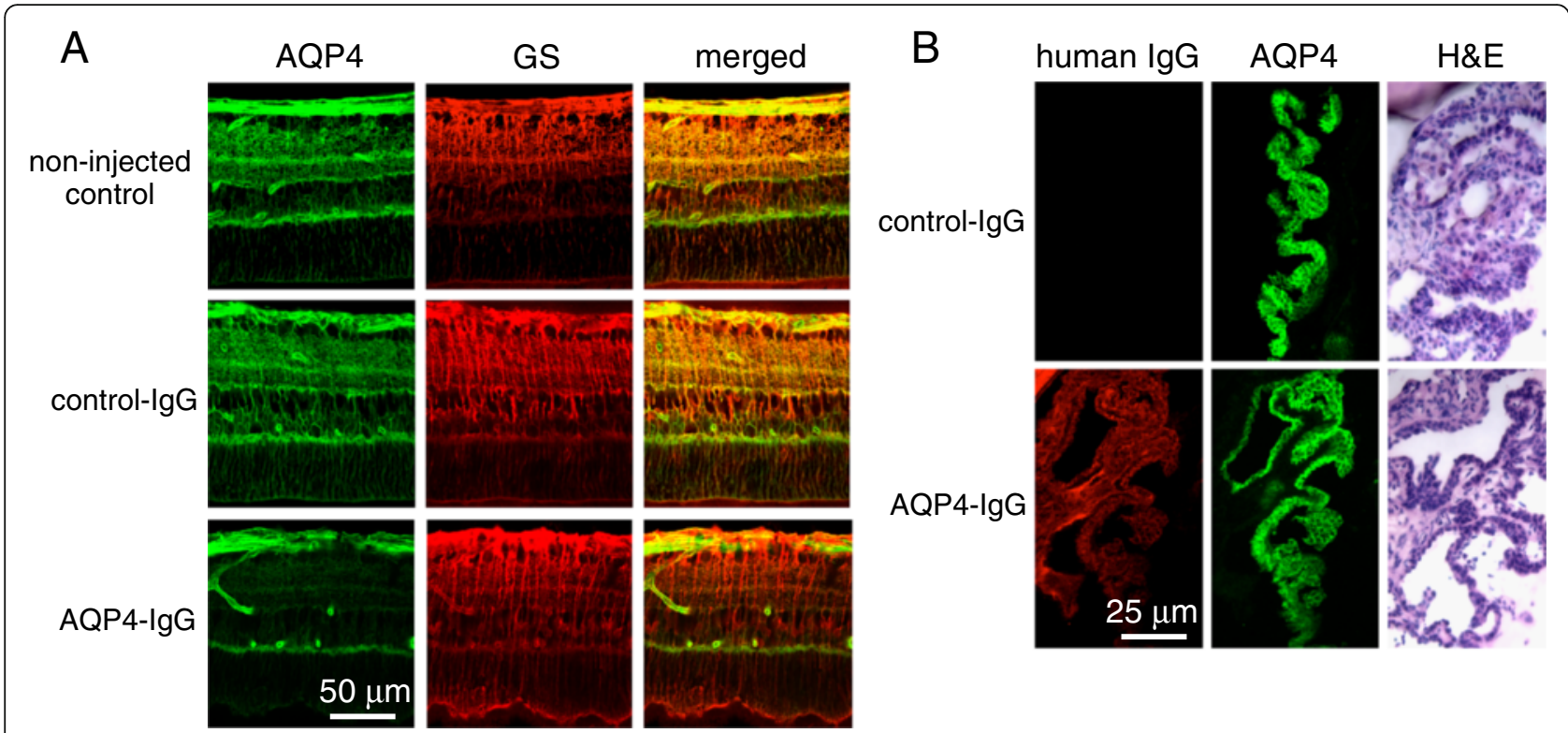

Fig. 2 Selective loss of Müller cell AQP4 following intravitreal injection of AQP4-lgG. a AQP4 and GS immunofluorescence at 5 days after intravitreal AQP4-lgG injection under conditions as in Fig. 1c, visualized by confocal microscopy using a $\times 60$ oil immersion lens. b Human lgG and AQP4 immunofluorescence and H\&E staining of the ciliary body at 5 days following intravitreal AQP4-lgG injection. Images representative of four eyes

exposure of the retinas of transgenic rats lacking the complement regulator protein CD59 to AQP4-IgG produced similar changes in AQP4 and GFAP immunofluorescence as in wildtype rats. The quantification of AQP4 and GFAP immunofluorescence is summarized in Fig. 3b.

To show that the loss of Müller cell AQP4 is specific to AQP4-IgG binding rather than a nonspecific effect of ocular inflammation, retinas were examined at 5 days after intravitreal delivery of LPS, a model that produces uveitis with vitritis and associated Müller cell gliosis [22]. Figure 3c shows preservation of Müller cell AQP4 despite inflammation with panuveitis and abundance of macrophages in the vitreous, Müller cell gliosis, and cellular infiltration (see below).

\section{Intravitreal AQP4-IgG produces retinal microglial activation with minimal leukocyte infiltration}

Iba1 is a marker of monocytes, including brain-derived microglia and infiltrative macrophages. Immunofluorescence showed a significant increase in the number of microglia throughout the inner retinal layers, especially in IPL, 5 days after intravitreal AQP4-IgG administration. Increased numbers of Iba $1^{+}$cells were also seen in CVF-treated rats, again supporting a complementindependent proinflammatory mechanism (Fig. 4a). As a positive control, intravitreal LPS increased the number of $\mathrm{Iba}^{+}$cells. Examination of individual cells at high magnification showed a characteristic stellate appearance indicative of microglial activation (Fig. 4b). Though resident microglia were activated by AQP4-IgG, comparatively few $\mathrm{CD} 45^{+}$leukocytes were seen in the retinas (Fig. 4a); as a control, abundant $\mathrm{CD} 45^{+}$infiltrates were seen in the retinas of the rats following intravitreal LPS. The bright green fluorescence at the bottom of the section (below the ONL) is due to nonspecific secondary antibody binding to the sclera, which occasionally remained attached to the retina during sectioning.

\section{AQP4-IgG produces loss of retinal ganglion cells}

Retinal structures were examined longitudinally by in vivo fundus imaging. Fundus photography did not reveal acute vascular or inflammatory pathology, including the absence of retinal vascular occlusions, retinitis, or papillitis (Fig. 5a). OCT showed inner retinal thickening in the absence of microcyst formation at day 5 at which maximal AQP4 loss and microglial activation occurs, with mild though significant GCC and total retinal thinning at day 30 (Fig. 5b, c). As GCC layer thinning is consistent with loss of RGCs and/or Müller cell somas in the INL, Brn3a ${ }^{+}$RGCs were quantified in whole retinal mounts at day 30 . RGC density was significantly reduced in the AQP4-IgG- compared with control-IgG-treated eyes (Fig. 5d).

\section{Mechanism of AQP4-IgG-induced loss of Müller cell AQP4 studied in retinal explant cultures}

Retinal explant cultures were used to investigate the mechanism of AQP4-IgG-induced AQP4 loss in Müller cells. Culture of untreated retinas in serumfree media enabled preservation of retinal structures and Müller cell AQP4 expression after $24 \mathrm{~h}$ in culture. 

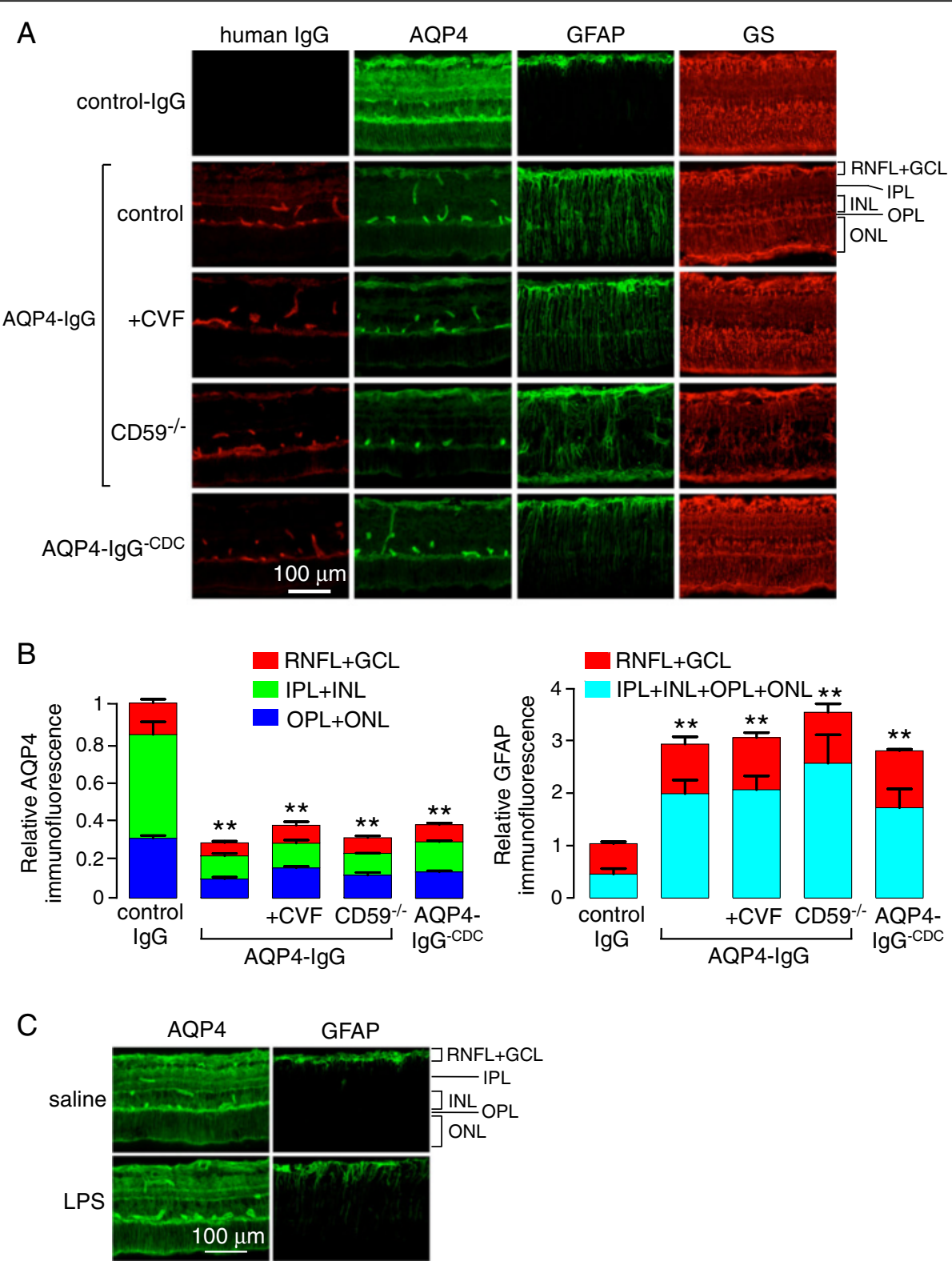

Fig. 3 Complement-independent loss of Müller cell AQP4 following intravitreal AQP4-lgG administration. a Immunofluorescence of the central retina at 5 days after intravitreal administration of AQP4-lgG (or control-lgG). Where indicated, rats were treated with cobra venom factor (CVF) to inactivate complement or administered AQP4-IgG ${ }^{-C D C}$ (AQP4-lgG lacking complement effector function) in place of AQP4-lgG. In some studies,

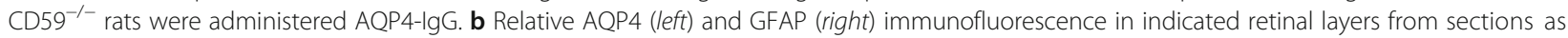
in (c) (mean \pm S.E.M, 2 rats, 4 eyes per condition, ${ }^{*} p<0.05,{ }^{* *} p<0.01$ ). c AQP4 and GFAP immunofluorescence at 5 days after intravitreal injection of saline control (top) or 5 $\mu \mathrm{g}$ LPS (bottom)

Subsequent incubation with AQP4-IgG, without added complement or leukocytes, produced marked, $\sim 50 \%$ reduction in Müller cell AQP4 expression $24 \mathrm{~h}$ later (Fig. 6a, b). To investigate whether an endocytosis mechanism might be responsible for the reduced AQP4 expression, the retinas were incubated during the 24-h exposure to AQP4-IgG with an inhibitor of clathrin-mediated endocytosis, dynasore, or an inhibitor of lysosomal acidification/degradation, chloroquine.
AQP4-IgG-induced AQP4 loss was largely prevented by dynasore or chloroquine (Fig. 6a, b). Highmagnification confocal microscopy of retinas at $4 \mathrm{~h}$ after addition of AQP4-IgG showed numerous AQP4 ${ }^{+}$ puncta that were not seen in the control retinas, supporting an endocytic retrieval mechanism. Together, these data support an endocytosis and lysosomal degradation mechanism for AQP4 loss on AQP4-IgGexposed Müller cells. 

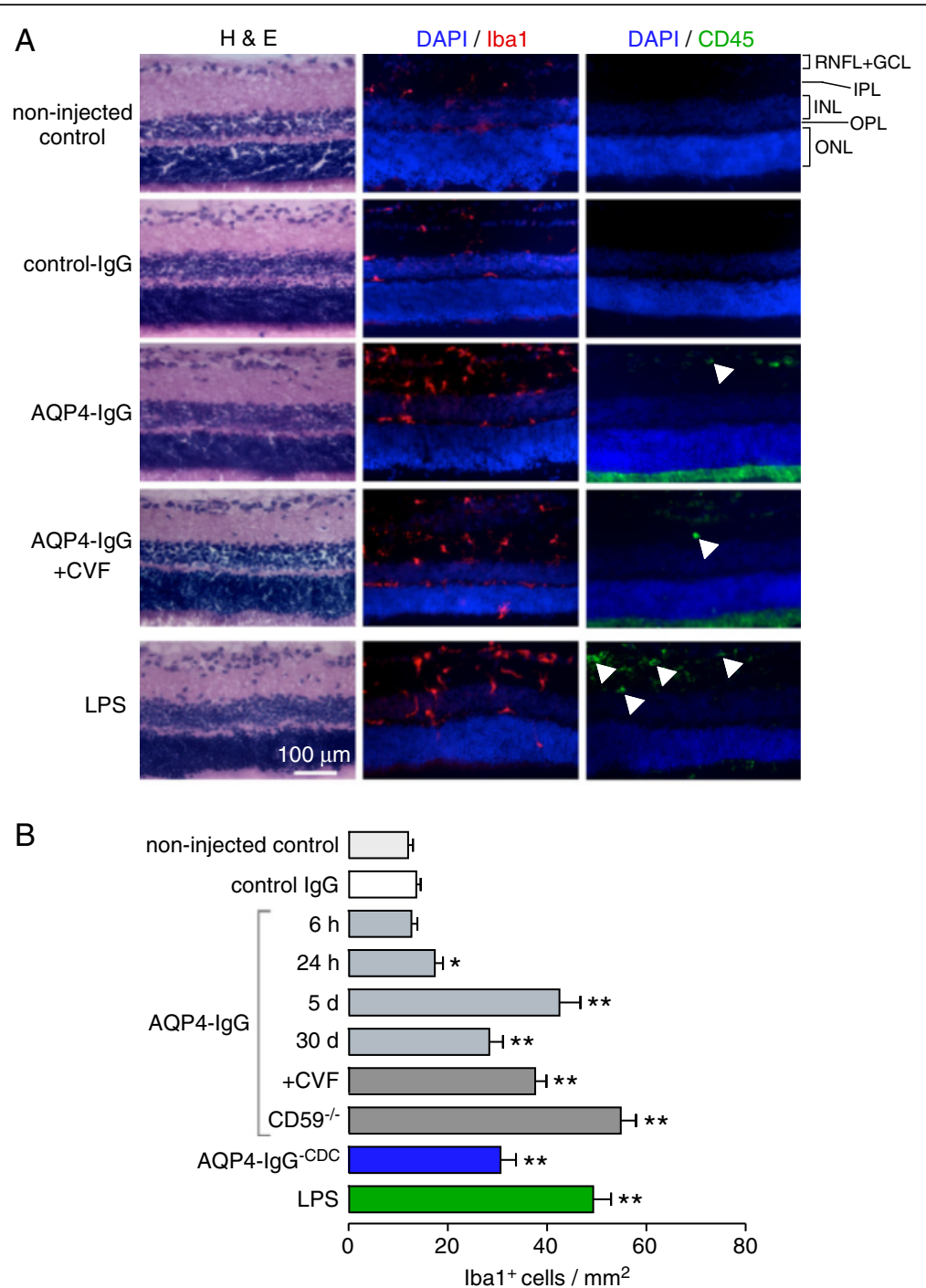

Fig. 4 Retinal inflammatory response to intravitreal AQP4-IgG. a H\&E staining, and Iba1 and CD45 immunofluorescence (with DAPI counterstain), in posterior retinas at 5 days after intravitreal injection of $40 \mu \mathrm{g} \mathrm{AQP4-IgG} \mathrm{(or} \mathrm{control-lgG).} \mathrm{White} \mathrm{arrowheads} \mathrm{indicate} \mathrm{CD45} 5^{+}$cells. $\mathbf{b} \mid \mathrm{lba} 1^{+}$cells per $\mathrm{mm}^{2}$ of full-thickness retina (mean \pm S.E.M, 2 rats, 4 eyes per condition, ${ }^{*} p<0.05,{ }^{* *} p<0.01$ )

\section{Discussion}

We found that exposure of retinal Müller cells to AQP4IgG can produce primary retinal pathology in the absence of NMO optic neuritis. For these studies, we administered a purified, recombinant AQP4-IgG to rat eyes by an intravitreal route. AQP4-IgG diffused through the posterior segment where it accessed the ciliary epithelium and retinal Müller cells, without significant diffusion to AQP4 on retrobulbar optic nerve astrocytes. We found that intravitreal injection of AQP4-IgG produced pathology with loss of Müller cell AQP4, a gliotic response with increased GFAP, microglial activation with minimal leukocyte infiltration, and mild RGC loss and thinning of the GCC. An interesting and unexpected observation was that the changes were complement independent, which contrasts with the generally accepted
NMO pathogenesis mechanism in the optic nerve, spinal cord, and brain. These findings have potential implications for pathogenesis mechanisms and therapy of retinal abnormalities in NMO.

The reduced Müller cell AQP4 expression following exposure to AQP4-IgG in ex vivo retinal cultures supports an endocytic retrieval mechanism with lysosomal targeting to account for the loss of AQP4. The comparable reduction in Müller cell AQP4 following intravitreal administration of an engineered AQP4-IgG lacking complement and cellular effector functions supports the conclusion that AQP4-IgG itself causes AQP4 internalization, as do the retinal culture studies in which neither complement nor effector cells were added. AQP4-IgGinduced internalization of AQP4 is likely cell-type-, polarization-, and, perhaps, even species-specific. While 

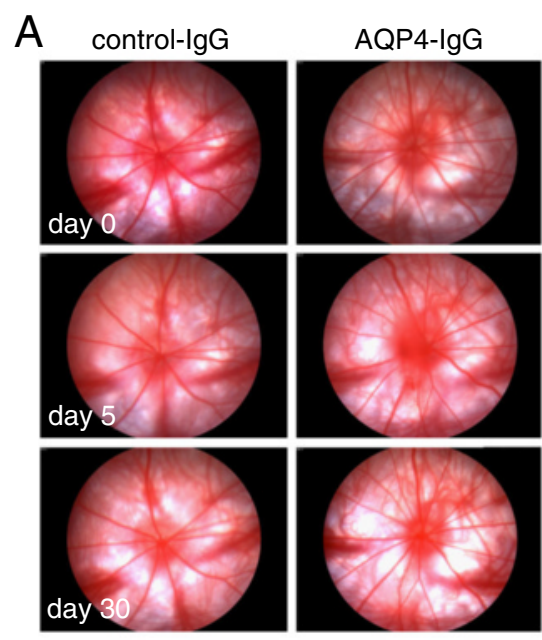

C
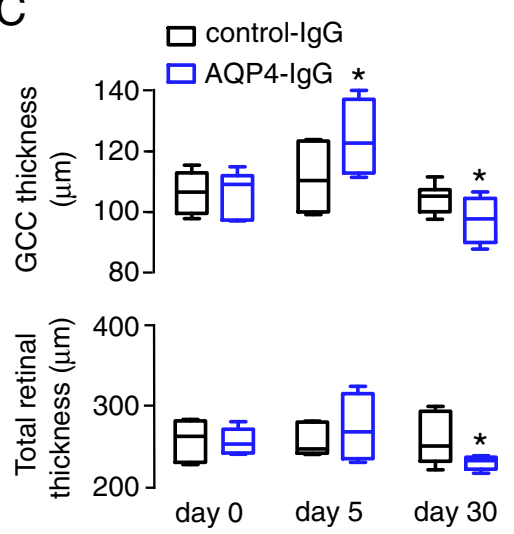

B
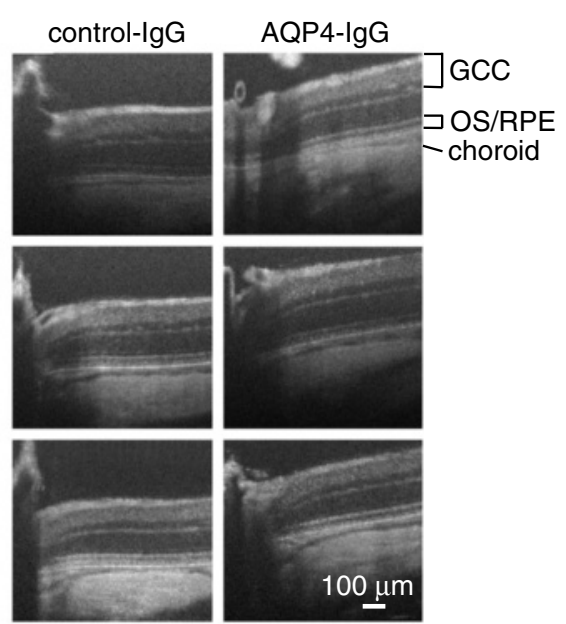

D control-lgG
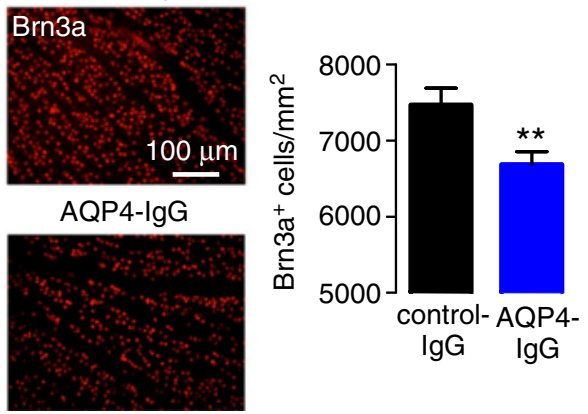

Fig. 5 Fundoscopy, OCT, and RGC count in rat eyes following intravitreal injection of AQP4-lgG. a Serial fundus photographs of individual rat eyes following intravitreal injection of AQP4-lgG (or control-lgG). b SD-OCT horizontal line scans through the optic nerve head in rat eyes for studies as in (a). OS/RPE outer segment/retinal pigment epithelium. c GCC (top) and total retinal (bottom) thicknesses before and at 5 and 30 days after intravitreal injection of AQP4-IgG (or control-lgG) (box and whisker plot with top line indicating maximum value, middle bar the mean, and lower line the minimum, mean \pm S.E.M, 3 rats, 6 eyes per condition, ${ }^{*} p<0.05,{ }^{* *} p<0.01$ ). $\mathbf{d}$ (Left) Brn3a immunofluorescence in mid-peripheral retina in flat mounts at 30 days after intravitreal injection of AQP4-IgG or control-lgG. (Right) Brn3a ${ }^{+}$RGC cell counts (mean \pm S.E.M, 2 rats, 4 eyes per condition, ${ }^{*} p<0.05$ )

AQP4-IgG produces rapid AQP4 internalization in several AQP4-transfected cell lines, and moderately fast AQP4 internalization in nonpolarized astrocyte primary cultures, little AQP4-IgG-dependent AQP4 internalization was seen in astrocytes in the mouse brain in vivo $[23,24]$. Though Müller cell AQP4 expression is polarized, the biology of Müller cells is different from that of astrocytes in the brain, spinal cord, and optic nerve. It is not clear from the studies here whether the loss of RGCs is a consequence of the loss of Müller cell AQP4 expression, the gliotic response, and/or intrinsic retinal inflammation.

The largely complement-independent retinal pathology following intravitreal AQP4-IgG administration contrasts with that seen in other CNS tissues. In humans, centrovascular deposition of activated complement is a characteristic pathological feature in NMO [25], and an initial open-label clinical study of a complement inhibitor showed efficacy in reducing attacks of NMO optic neuritis and transverse myelitis in seropositive NMO patients [26]. Pathology following passive transfer of AQP4-IgG to the mouse and rat brain, optic nerve, and spinal cord is predominantly complement dependent, as omission of added complement to mice [27-29] or inactivation of endogenous complement in rats $[18,19]$ prevents the development of NMO pathology. The complement-independent retinal injury found here suggests that complement-targeted therapeutics may have limited efficacy in some NMO patients with anterior visual pathway involvement.

Another interesting observation was increased GFAP expression in Müller cells following intravitreal AQP4- 

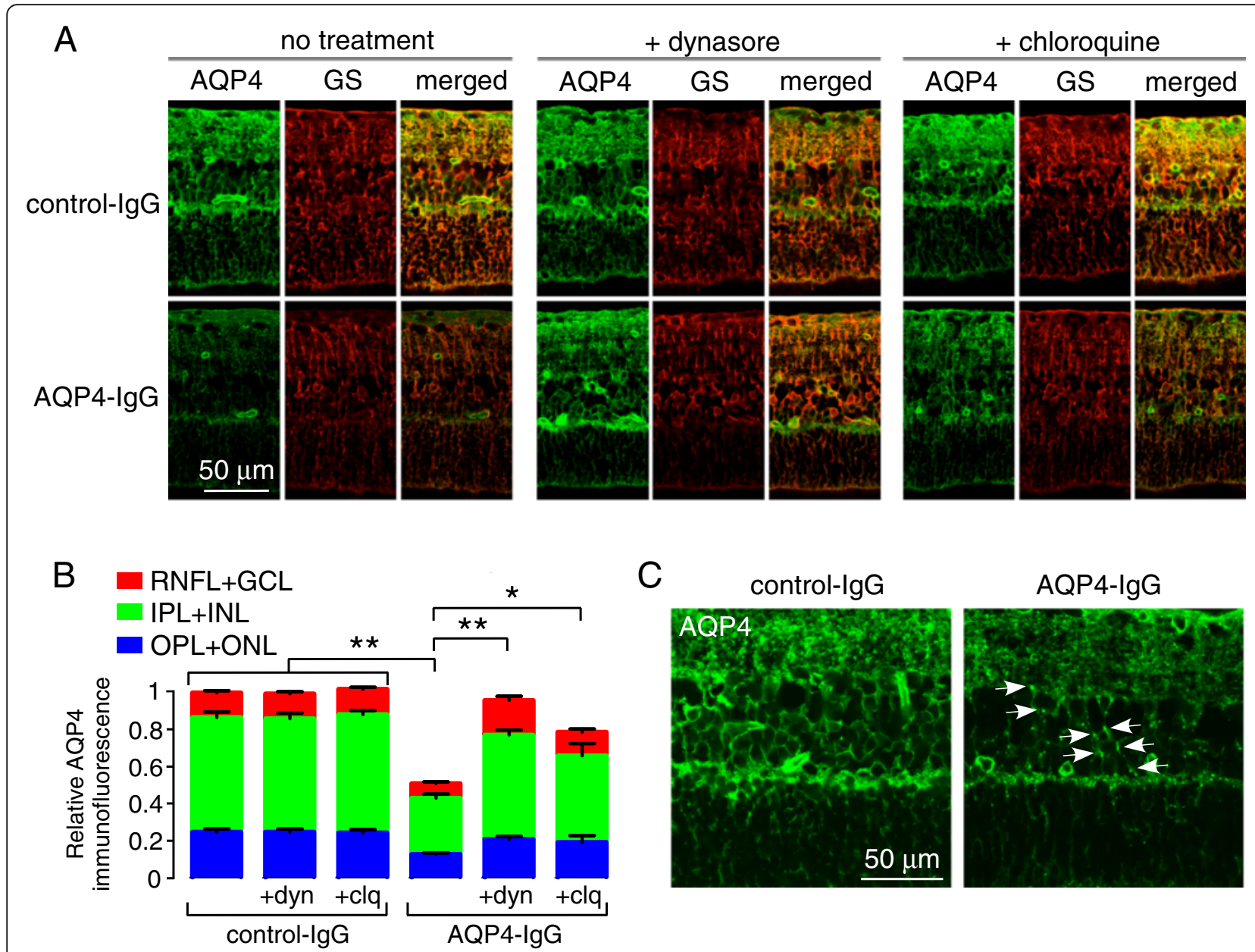

C
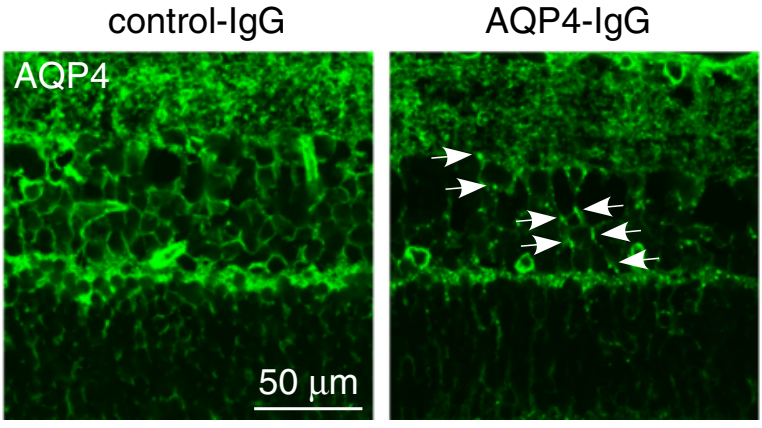

Fig. 6 AQP4-lgG reduces Müller cell AQP4 expression in retinal explant cultures by an endocytosis mechanism. a AQP4 and GS immunofluorescence in retinal explant cultures treated with $20 \mu \mathrm{g} / \mathrm{mL}$ AQP4-lgG (or control-lgG) for $24 \mathrm{~h}$, in the absence or presence of $50 \mu \mathrm{M}$ dynasore hydrate (dyn) or $10 \mu \mathrm{M}$ chloroquine (clq). b Relative AQP4 immunofluorescence for experiments as in (a) (mean \pm S.E.M, $n=6,{ }^{*} p<0.05$, ${ }^{* *} p<0.01$ ). c AQP4 immunofluorescence at high magnification for explant cultures treated for $4 \mathrm{~h}$ with AQP4-lgG (or control-lgG). White arrows indicate endocytic vesicles

IgG administration, indicating a prominent gliotic response. Müller cell gliosis may be a consequence of intraretinal inflammation, Müller cell injury, and/or AQP4 loss. The retinal pathology seen here of AQP4 loss with increased GFAP and with minimal early complement deposition corresponds to the pathology seen in active human NMO lesions found in the brain and spinal cord classified as type 4 pathology [30]. Similarly, two separate models of chronic intrathecal AQP4-IgG infusion have shown loss of AQP4 expression in the spinal cord with reactive gliosis in the absence of complement activation [31, 32]. Thus, under conditions in which AQP4-IgG does not activate complement, the astrocytic response can be associated with GFAP upregulation rather than downregulation as seen when complement is activated. Release of soluble factors by activated microglia and Müller cells such as the proinflammatory cytokines TNF and monocyte chemoattractant protein $\mathrm{MCP}-1$, which are thought to contribute to retinal degeneration in diabetic and other retinopathies [33], may contribute to the retinal pathology. Chronic functional loss of AQP4 water permeability in Müller cells was found in AQP4 knockout mice to produce abnormalities in retinal signal transduction as seen by electroretinography [34], which may reflect abnormal potassium and glutamate homeostasis. It is not clear whether the Müller cell gliosis and RGC loss produced by intravitreal AQP4-IgG is due to acute and partial loss of Müller cell water-transporting function.

Though the AQP4-expressing ciliary epithelium strongly bound AQP4-IgG following intravitreal AQP4IgG administration, no pathology was seen at the light microscopic level. This is consistent with the near absence, albeit for a few case reports of NMO-related myositis [35, 36], of NMO pathology in peripheral organs in which AQP4 is expressed, including the 
skeletal muscle, stomach, kidney, airways, and exocrine glands. High-dose systemic administration of AQP4-IgG to mice or rats produces prompt and extensive AQP4IgG deposition on peripheral AQP4-expressing organs, but with no pathology [18, 37]. Humans have been reported with circulating AQP4-IgG for at least a decade preceding clinical NMO disease [38]. Why peripheral, AQP4-expressing tissues are not damaged in seropositive NMO, despite their exposure to AQP4-IgG, remains unclear. It has been speculated that the unique cellular and physical environment in the CNS may be responsible for the development of NMO pathology in CNS but not peripheral tissues, as might the presence of complement inhibitor or other anti-inflammatory mechanisms in peripheral tissues.

During the completion of our manuscript, a paper by Zeka et al. [39] was published reporting that rats receiving AQP4-specific T cells developed retinitis with $\mathrm{T}$ cell infiltration and axonal pathology and that loss of AQP4 on Müller cells was seen when AQP4-IgG was delivered systemically along with the AQP4-specific $\mathrm{T}$ cells. Whether the retinal changes were primary or secondary to optic nerve pathology is unclear, as is its relevance to retinal abnormalities in human NMO.

Though our study demonstrates that exposure of the retina to AQP4-IgG can produce primary retinal injury with associated RGC loss, it is not known whether such a mechanism occurs in human NMO. Whether AQP4IgG can access and bind to AQP4 on Müller cells beyond the blood-retinal barrier has not been established. There are no reports to our knowledge of retinal abnormalities in NMO without a history of optic neuritis, making it difficult to resolve primary vs. secondary AQP4-IgG-induced retinal injury in human NMO. Retinal pathology has been identified in postmortem tissue of patients with NMO, with mild loss of calbindinpositive horizontal cells and moderate loss of Müller cells and scattered loss of AQP4 immunoreactivity [40]. There were also Iba $1^{+}$microglia in the inner retina with few $\mathrm{CD} 45^{+}$cells and little complement deposition. Our animal model recapitulates many of these features of retinal pathology in human NMO.

\section{Conclusions}

Passive transfer of NMO anti-AQP4 autoantibody by intravitreal injection in rat eyes produced marked loss of Müller cell AQP4 expression with a gliotic response, microglial activation, and mild RGC loss. The action of the autoantibody was largely complement independent, which contrasts with NMO pathogenesis mechanisms in the brain, spinal cord, and optic nerve. The results here provide a potential explanation and mechanism for the retinal pathology seen in seropositive NMO.

\begin{abstract}
Abbreviations
AQP4: Aquaporin-4; AQP4-IgG: anti-AQP4 autoantibody;

AQP4-IgG ${ }^{-C D C}$ : Aquaporumab, AQP4-lgG-lacking effector functions; clq: Chloroquine; CNS: Central nervous system; control-lgG: Control human IgG; CVF: Cobra venom factor; dyn: Dynasore hydrate; GCC: Ganglion cell complex; GCL: Ganglion cell layer; GFAP: Glial fibrillary acidic protein; GS: Glutamine synthetase; H\&E: Hematoxylin and eosin; HBSS: Hank's balanced salt solution; Iba1: Ionized calcium-binding adaptor molecule-1; INL: Inner nuclear layer; IPL: Inner plexiform layer; LPS: Lipopolysaccharide; Neurobasal A: Neuronal growth medium; NMO: Neuromyelitis optica; ONL: Outer nuclear layer; OPL: Outer plexiform layer; PBS: Phosphate-buffered saline; RGCs: Retinal ganglion cells; RNFL: Retinal nerve fiber layer; SD-OCT: Spectral-domain optical coherence tomography
\end{abstract}

\section{Acknowledgements}

We thank Dr. Jeff Bennett (U. Colorado) for providing rAb-53 antibody, Dr. Xiaoming Yao (UCSF) for providing $\mathrm{CD} 59^{-/-}$rats, and Hao Yiu and Dr. Marcel Alavi (UCSF) for their assistance in the acquisition and analysis of the OCT data.

\section{Funding}

This project was supported by a Research to Prevent Blindness Career Development Award, a National Institutes of Health Grant (EY023981), a UCSF Clinical and Translational Science Institute award, and That Man May See (to MHL), National Institutes of Health Grants (EY13574, DK72517, EB00415, DK35124, and DK101373) (to ASV), and a National Eye Institute Core Grant for Vision Research (EY002162) and Unrestricted Grant from Research to Prevent Blindness to the UCSF Department of Ophthalmology.

\section{Availability of data and materials}

All data supporting the conclusions of this manuscript are shown in the text and figures.

\section{Authors' contributions}

CMF performed the experiments and analyzed the data. CMF, MHL, and ASV participated in the conception of the study and designed the experiments. All authors contributed to the writing and editing and approved the final manuscript.

\section{Competing interests}

The authors declare that they have no competing interests.

\section{Consent for publication}

Not applicable.

\section{Ethics approval and consent to participate}

All procedures were approved by the University of California San Francisco Committee on Animal Research and were in compliance with the ARVO

Statement for the Use of Animals in Ophthalmic and Visual Research.

Consent to participate is not applicable.

\section{Author details}

'Department of Ophthalmology, University of California, San Francisco, San Francisco, CA, USA. ${ }^{2}$ Departments of Medicine and Physiology, University of California, San Francisco, 1246 Health Sciences East Tower, San Francisco, CA 94143-0521, USA. ${ }^{3}$ Department of Ophthalmology, The Palo Alto Medical Foundation, Palo Alto, CA, USA.

Received: 20 August 2016 Accepted: 13 October 2016

Published online: 20 October 2016

\section{References}

1. Hinson SR, Lennon VA, Pittock SJ. Autoimmune AQP4 channelopathies and neuromyelitis optica spectrum disorders. Handb Clin Neurol. 2016; 133:377-403. doi:10.1016/B978-0-444-63432-0.00021-9.

2. Bennett JL, de Seze J, Lana-Peixoto M, Palace J, Waldman A, Schippling S, Tenembaum S, Banwell B, Greenberg B, Levy M, Fujihara K, Chan KH, Kim HJ, Asgari N, Sato DK, Saiz A, Wuerfel J, Zimmermann H, Green A, Villoslada P, Paul F, GJCF-ICC\&BR. Neuromyelitis optica and multiple sclerosis: seeing differences through optical coherence tomography. Mult Scler. 2015;21(6): 678-88. doi:10.1177/1352458514567216. 
3. Papadopoulos MC, Bennett JL, Verkman AS. Treatment of neuromyelitis optica: state-of-the-art and emerging therapies. Nat Rev Neurol. 2014;10: 493-506. doi:10.1038/nrneurol.2014.141.

4. Pittock SJ, Lucchinetti CF. Neuromyelitis optica and the evolving spectrum of autoimmune aquaporin-4 channelopathies: a decade later. Ann N Y Acad Sci. 2016;1366:20-39. doi:10.1111/nyas.12794.

5. Merle H, Olindo S, Donnio A, Richer R, Smadja D, Cabre P. Retinal peripapillary nerve fiber layer thickness in neuromyelitis optica. Invest Ophthalmol Vis Sci. 2008;49:4412-7. doi:10.1167/iovs.08-1815.

6. Ratchford JN, Quigg ME, Conger A, Frohman T, Frohman E, Balcer LJ, Calabresi PA, Kerr DA. Optical coherence tomography helps differentiate neuromyelitis optica and MS optic neuropathies. Neurology. 2009;73:302-8. doi:10.1212/WNL.0b013e3181af78b8.

7. Naismith RT, Tutlam NT, Xu J, Klawiter EC, Shepherd J, Trinkaus K, Song SK, Cross AH. Optical coherence tomography differs in neuromyelitis optica compared with multiple sclerosis. Neurology. 2009:72:1077-82. doi:10.1212/01.wnl.0000345042.53843.d5.

8. Fernandes DB, Raza AS, Nogueira RGF, Wang D, Callegaro D, Hood DC, Monteiro MLR. Evaluation of inner retinal layers in patients with multiple sclerosis or neuromyelitis optica using optical coherence tomography. Ophthalmology. 2013:120:387-94. doi:10.1016/j.ophtha.2012.07.066.

9. Monteiro MLR, Fernandes DB, Apóstolos-Pereira SL, Callegaro D. Quantification of retinal neural loss in patients with neuromyelitis optica and multiple sclerosis with or without optic neuritis using Fourier-domain optical coherence tomography. Investig Opthalmology Vis Sci. 2012;53:3959. doi:10.1167/iovs.11-9324

10. Gelfand JM, Cree BA, Nolan R, Arnow S, Green AJ. Microcystic inner nuclear layer abnormalities and neuromyelitis optica. JAMA Neurol. 2013;70:629-33. doi:10.1001/jamaneurol.2013.1832.

11. Sotirchos ES, Saidha S, Byraiah G, Mealy MA, Ibrahim MA, Sepah YJ, Newsome SD, Ratchford JN, Frohman EM, Balcer LJ, Crainiceanu CM, Nguyen QD, Levy M, Calabresi PA. In vivo identification of morphologic retinal abnormalities in neuromyelitis optica. Neurology. 2013;80:1406-14. doi:10.1212/WNL.0b013e31828c2f7a.

12. Pott JWR, de Vries-Knoppert WAEJ, Petzold A. The prevalence of microcystic macular changes on optical coherence tomography of the macular region in optic nerve atrophy of non-neuritis origin: a prospective study. $\mathrm{Br} J$ Ophthalmol. 2016;100:216-21. doi:10.1136/bjophthalmol-2014-305737.

13. Hasegawa T, Akagi T, Yoshikawa M, Suda K, Yamada H, Kimura Y, Nakanishi $\mathrm{H}$, Miyake M, Unoki N, Ikeda HO, Yoshimura N. Microcystic inner nuclear layer changes and retinal nerve fiber layer defects in eyes with glaucoma. PLoS One. 2015;10(6). e013175.

14. Saidha S, Sotirchos ES, Ibrahim MA, Crainiceanu CM, Gelfand JM, Sepah YJ, Ratchford JN, Oh J, Seigo MA, Newsome SD, Balcer L, Frohman EM, Green AJ, Nguyen QD, Calabresi PA. Microcystic macular oedema, thickness of the inner nuclear layer of the retina, and disease characteristics in multiple sclerosis: a retrospective study. Lancet Neurol. 2012;11:963-72. doi:10.1016/ S1474-4422(12)70213-2.

15. Barboni P, Carelli V, Savini G, Carbonelli M, La Morgia C, Sadun AA. Microcystic macular degeneration from optic neuropathy: not inflammatory, not trans-synaptic degeneration. Brain. 2013;136(Pt 7):e239. doi:10.1093/ brain/awt014

16. Levin $\mathrm{MH}$, Bennett JL, Verkman AS. Optic neuritis in neuromyelitis optica. Prog Retin Eye Res. 2013;36:159-71. doi:10.1016/j.preteyeres.2013.03.001.

17. Ratelade J, Verkman AS. Inhibitor(s) of the classical complement pathway in mouse serum limit the utility of mice as experimental models of neuromyelitis optica. Mol Immunol. 2014;62:104-13. doi:10.1016/j.molimm.2014.06.003.

18. Asavapanumas N, Verkman AS. Neuromyelitis optica pathology in rats following intraperitoneal injection of NMO-lgG and intracerebral needle injury. Acta Neuropathol Commun. 2014;2:48. doi:10.1186/2051-5960-2-48.

19. Asavapanumas N, Ratelade J, Verkman AS. Unique neuromyelitis optica pathology produced in naïve rats by intracerebral administration of $\mathrm{NMO}$ IgG. Acta Neuropathol. 2014;127:539-51. doi:10.1007/s00401-013-1204-8.

20. Bennett JL, Lam C, Kalluri SR, Saikali P, Bautista K, Dupree C, Glogowska M, Case D, Antel JP, Owens GP, Gilden D, Nessler S, Stadelmann C, Hemmer B. Intrathecal pathogenic anti-aquaporin-4 antibodies in early neuromyelitis optica. Ann Neurol. 2009;66:617-29. doi:10.1002/ana.21802.

21. Tradtrantip L, Zhang H, Saadoun S, Phuan PW, Lam C, Papadopoulos MC, Bennett JL, Verkman AS. Anti-aquaporin-4 monoclonal antibody blocker therapy for neuromyelitis optica. Ann Neurol. 2012;71:314-22. doi:10.1002/ana.22657.
22. Liu XQ, Kobayashi H, Jin ZB, Wada A, Nao-I N. Differential expression of Kir4. 1 and aquaporin 4 in the retina from endotoxin-induced uveitis rat. Mol Vis. 2007:13:309-17.

23. Ratelade J, Bennett $J$, Verkman AS. Intravenous neuromyelitis optica autoantibody in mice targets aquaporin-4 in peripheral organs and area postrema. PLoS One. 2011;6:e27412. doi:10.1371/journal.pone. 0027412.

24. Rossi A, Ratelade J, Papadopoulos MC, Bennett JL, Verkman AS. Neuromyelitis optica lgG does not alter aquaporin-4 water permeability, plasma membrane M1/M23 isoform content, or supramolecular assembly. Glia. 2012;60:2027-39. doi:10.1002/glia.22417.

25. Papadopoulos MC, Verkman AS. Aquaporin 4 and neuromyelitis optica. Lancet Neurol. 2012;11:535-44. doi:10.1016/S1474-4422(12)70133-3.

26. Pittock SJ, Lennon VA, McKeon A, Mandrekar J, Weinshenker BG, Lucchinetti CF, O'Toole O, Wingerchuk DM. Eculizumab in AQP4-lgGpositive relapsing neuromyelitis optica spectrum disorders: an open-label pilot study. Lancet Neurol. 2013;12:554-62. doi:10.1016/ S1474-4422(13)70076-0

27. Asavapanumas N, Ratelade J, Papadopoulos MC, Bennett JL, Levin MH, Verkman AS. Experimental mouse model of optic neuritis with inflammatory demyelination produced by passive transfer of neuromyelitis opticaimmunoglobulin G. J Neuroinflammation. 2014;11:16. doi:10.1186/17422094-11-16.

28. Saadoun S, Waters P, Bell BA, Vincent A, Verkman AS, Papadopoulos MC Intra-cerebral injection of neuromyelitis optica immunoglobulin $\mathrm{G}$ and human complement produces neuromyelitis optica lesions in mice. Brain 2010;133:349-61. doi:10.1093/brain/awp309.

29. Zhang $H$, Verkman AS. Longitudinally extensive NMO spinal cord pathology produced by passive transfer of NMO-lgG in mice lacking complement inhibitor CD59. J Autoimmun. 2014;53:67-77. doi:10.1016/ j.jaut.2014.02.011

30. Misu T, Höftberger R, Fujihara K, Wimmer I, Takai Y, Nishiyama S, Nakashima l, Konno H, Bradl M, Garzuly F, Itoyama Y, Aoki M, Lassmann H. Presence of six different lesion types suggests diverse mechanisms of tissue injury in neuromyelitis optica. Acta Neuropathol. 2013;125:815-27. doi:10.1007/s00401-013-1116-7.

31. Marignier R, Ruiz A, Cavagna S, Nicole A, Watrin C, Touret M, Parrot S, Malleret G, Peyron C, Benetollo C, Auvergnon N, Vukusic S, Giraudon P. Neuromyelitis optica study model based on chronic infusion of autoantibodies in rat cerebrospinal fluid. J Neuroinflammation. 2016;13:111. doi:10.1186/s12974-016-0577-8.

32. Geis C, Ritter C, Ruschil C, Weishaupt A, Grünewald B, Stoll G, Holmoy T, Misu T, Fujihara K, Hemmer B, Stadelmann C, Bennett J, Sommer C, Toykaa $\mathrm{KV}$. The intrinsic pathogenic role of autoantibodies to aquaporin 4 mediating spinal cord disease in a rat passive-transfer model. Exp Neurol. 2015;265:8-21. doi:10.1016/j.expneurol.2014.12.015.

33. Bringmann A, landiev I, Pannicke T, Wurm A, Hollborn M, Wiedemann P, Osborne NN, Reichenbach A. Cellular signaling and factors involved in Müller cell gliosis: neuroprotective and detrimental effects. Prog Retin Eye Res. 2009;28:423-51. doi:10.1016/j.preteyeres.2009.07.001

34. Li J, Patil RV, Verkman AS. Mildly abnormal retinal function in transgenic mice without Müller cell aquaporin-4 water channels. Invest Ophthalmol Vis Sci. 2002;43:573-9.

35. Deguchi S, Deguchi K, Sato K, Yunoki T, Omote Y, Morimoto N, Kurata T, Ikeda M, Takahashi T, Aoki M, Abe K. HyperCKemia related to the initial and recurrent attacks of neuromyelitis optica. Intern Med. 2012;51: 2617-20.

36. Malik R, Lewis A, Cree BAC, Ratelade J, Rossi A, Verkman AS, Bollen AW, Ralph JW. Transient hyperckemia in the setting of neuromyelitis optica (NMO). Muscle Nerve. 2014:50:859-62. doi:10.1002/mus.24298.

37. Ratelade J, Zhang H, Saadoun S, Bennett JL, Papadopoulos MC, Verkman AS Neuromyelitis optica lgG and natural killer cells produce NMO lesions in mice without myelin loss. Acta Neuropathol. 2012;123:861-72. doi:10.1007/ s00401-012-0986-4.

38. Nishiyama S, Ito T, Misu T, Takahashi T, Kikuchi A, Suzuki N, Jin K, Aoki M, Fujihara K, Itoyama Y. A case of NMO seropositive for aquaporin-4 antibody more than 10 years before onset. Neurology. 2009;72:1960-1. doi:10.1212/ WNL.0b013e3181a82621.

39. Zeka B, Hastermann M, Kaufmann N, Schanda K, Pende M, Misu T, Rommer P, Fujihara K, Nakashima I, Dahle C, Leutmezer F, Reindl M, Lassmann H, Bradl M. Aquaporin 4-specific T cells and NMO-lgG cause primary retinal 
damage in experimental NMO/SD. Acta Neuropathol Commun. 2016;4:82. doi:10.1186/s40478-016-0355-y.

40. Hokari M, Yokoseki A, Arakawa M, Saji E, Yanagawa K, Yanagimura F, Toyoshima Y, Okamoto K, Ueki S, Hatase T, Ohashi R, Fukuchi T, Akazawa K, Yamada M, Kakita A, Takahashi H, Nishizawa M, Kawachi I. Clinicopathological features in anterior visual pathway in neuromyelitis optica. Ann Neurol. 2016;79(4):605-24. doi:10.1002/ ana.24608.

Submit your next manuscript to BioMed Central and we will help you at every step:

- We accept pre-submission inquiries

- Our selector tool helps you to find the most relevant journal

- We provide round the clock customer support

- Convenient online submission

- Thorough peer review

- Inclusion in PubMed and all major indexing services

- Maximum visibility for your research

Submit your manuscript at www.biomedcentral.com/submit 